\title{
Freie Form - experimentelle Tragstruktur
}

\section{Entwurf und Umsetzung von modularen Flächentragwerken}

In dem Studentenprojekt „Experimental Structures“ wurden im Sommersemester 2014 am Lehrstuhl für Tragwerksplanung der Technischen Universität München sieben Tragstrukturen auf experimentelle Weise entwickelt. Die Masterstudenten nutzten Techniken wie Flechten, Falten oder Biegen, um parametrisch kontrollierte Strukturen auf modularer Basis zu erstellen. Dabei wurden unterschiedliche Lösungsansätze für die Unterteilung und Konstruktion von gekrümmten Flächen aufgezeigt. Die Ergebnisse bewegen sich zwischen effizientem Tragwerk und spielerischer Skulptur. Die Strukturen von ca. $2 \times 2$ m Spannweite wurden am Technischen Zentrum der TU mithilfe computergesteuerter Werkzeuge gefertigt und im Rahmen der Jahresschau der Architekturfakultät ausgestellt.

Keywords Entwerfen, parametrisches; Tragwerke, experimentelle; Tragwerke, elastisch gebogene; Tensegrity; Membrane; Faltstrukturen; Flechtstrukturen; CNC-Werkzeuge

\section{$1 \quad$ Aufgabenstellung}

Das Projekt „Experimental Structures“ entstand im Sommersemester 2014 am Lehrstuhl für Tragwerksplanung der Architekturfakultät der Technischen Universität München. Sieben ambitionierte Masterstudenten nahmen an dem einsemestrigen Entwurfsprojekt teil. Ziel war es,
Free form - experimental structure - design and realisation of modular surface structures

During the summer project 2014 "Experimental Structures" seven structures were developed through experimental trials at the Department for Structural Design at the Technical University in Munich. The master students utilized various techniques like braiding, folding or bending to generate parametrically controlled modular structures. This produced different approaches of tessellating and constructing double curved surfaces. The resulting works cover a range between efficient structures and playful sculptures. The structures of approximately $2 \times 2 \mathrm{~m}$ span were produced at the Technical Center of the TU using computer aided tools. They were exhibited at the Annual Show of the Architecture Faculty.

Keywords parametric design; experimental structures; elastic bending; tensegrity; membrane; folding; braiding; CNC tools

durch experimentelle Modellversuche neue Möglichkeiten für eine intelligente Symbiose von Formgebung, Herstellung und Tragwirkung aufzuzeigen.

Im Gegensatz zu üblichen Entwurfsprojekten an der Architekturfakultät wurden den Studenten zu Beginn des Kurses keine Vorgaben hinsichtlich Bauplatz, Nutzung

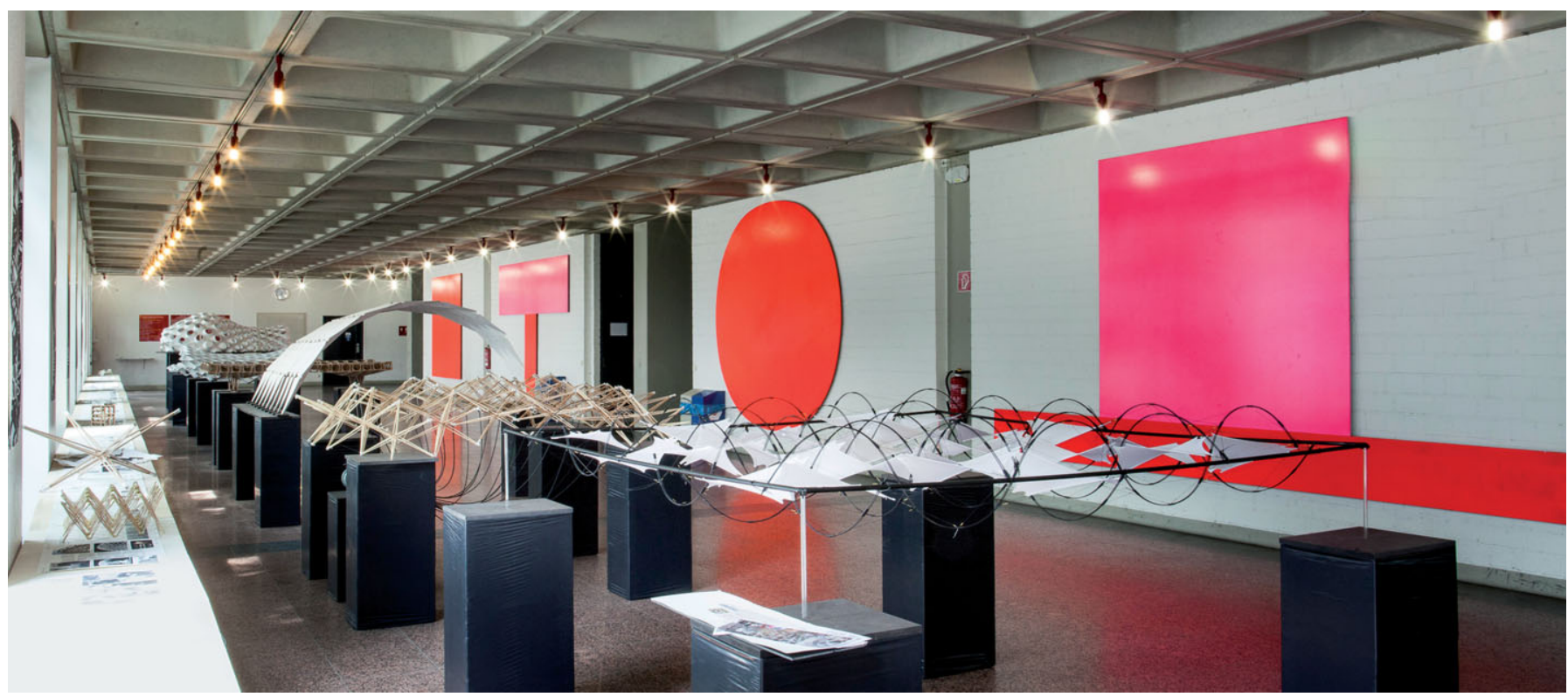

Bild 1 Ausstellung im Theresianum der Technischen Universität München Exhibtion at the Theresianum at the Technichal University in Munich 
oder Funktion ihres Entwurfes gemacht. Die Aufgabenstellung bestand lediglich darin, ein modulares Flächentragwerk zu entwickeln und mit einer Spannweite von ca. $2 \times 2 \mathrm{~m}$ zu bauen. Die Ergebnisse wurden im Rahmen der fakultären Jahresschau 2014 öffentlich ausgestellt (Bild 1).

\section{2}

\section{Entwurfsprozess}

$\mathrm{Zu}$ Beginn stand die ausführliche Recherche existierender Tragstrukturen. Es wurden unterschiedliche Versuchsgebiete festgelegt: Falt- und Flechtstrukturen, Tensegrity, Membrane und elastisch gebogene Strukturen. Die Studenten nutzten eine oder mehrere Techniken, um in Modellversuchen die Verhaltensweisen der Materialien $\mathrm{zu}$ testen und durch gezielte Verformung räumliche Tragmodule $\mathrm{zu}$ schaffen. Diese Einheiten wurden dann zu einem flächigen Tragsystem verknüpft. Dabei spielte die Ausbildung der Verbindungsdetails eine wichtige Rolle, da hiervon das Tragverhalten und die Form der Gesamtstruktur maßgeblich beeinflusst wurden.

Durch den „von innen nach außen“ formulierten Entwurfsprozess entstanden systemeigene Freiflächen, die nicht einer vorgegebenen Form, sondern der Logik ihrer Konstruktionsweise folgen.

\section{3}

\section{Parameterstudie}

Das grundsätzliche Verständnis aller Systemparameter galt als Voraussetzung für die Systementwicklung. Die Abhängigkeit zwischen Modulgeometrie und Gesamtsystem wurde in einer Parameterstudie nachvollzogen. Die einzelnen Parameter wurden bewertet und als veränderlich oder konstant definiert. Dadurch konnte der Planungsprozess fokussiert und eine gezielte Optimierung der Konstruktion ermöglicht werden.

Die Studenten wendeten diese Systematik entweder digital am Computer an oder nutzten das gewonnene Wissen für die Weiterentwicklung ihrer Prototypen.

\section{$4 \quad$ Verbindungen und Tragverhalten}

Der Modul- und Systementwicklung folgte eine Analyse der konstruktiven Verbindungen. Es wurde nach einfachen, kostengünstigen Lösungen gesucht, um die auftretenden Beanspruchungen - Druck, Zug, Biegung, Querkraft und Torsion - sowohl innerhalb des Moduls als auch modulübergreifend zu übertragen.

Im Rahmen des Projektes wurde das Tragverhalten unter Eigengewicht für jedes System qualitativ analysiert. Das so gewonnene Verständnis für die systemeigene Tragwirkung ermöglichte eine gezielte Weiterentwicklung von Geometrie und Konstruktion.
Die Realisierung des Tragsystems als eine ca. $2 \times 2 \mathrm{~m}$ große Skulptur hatte von Anfang an einen großen Einfluss auf den Entwurfsprozess und die Detailentwicklung. Die Studenten übernahmen die Verantwortung für eine ganzheitliche Planung inklusive Materialkosten und Beschaffung, Herstellung, Montage und Terminierung.

Es wurde eine Minimierung der Baukosten durch die intelligente Anwendung von einfachen Verbindungsmitteln und Materialien angestrebt. Gleichzeitig ermöglichten die computergesteuerten Werkzeuge des Technischen Zentrums den Studenten eine präzise und effiziente Fertigung.

\section{Ergebnisse}

An den Ergebnissen ist die für Architekten ungewöhnliche Aufgabenstellung eines rein technischen Entwurfes eindrucksvoll ablesbar. Durch bewusstes Ausklammern architektonischer Vorgaben konnten sich die Studenten ganz auf das Tragkonzept und dessen konstruktive Ausformung konzentrieren. Es entstanden ungewöhnliche Tragsysteme, die zum Nachdenken anregen. Die Strukturen bewegen sich in einem Spannungsfeld zwischen Tragwerk und Skulptur, Klarheit und Komplexität, Wiederholung und Veränderung, Konstruktion und Design.

Die sieben Arbeiten werden im Folgenden einzeln vorgestellt. Sie lassen sich nach den geometrischen Eigenschaften des Gesamtsystems in drei Gruppen gliedern:

Die erste Gruppe (Abschn. 6.1, 6.2, 6.3) beinhaltet eine planare und zwei leicht gekrümmte Systeme aus identischen Modulen. Die folgenden zwei Strukturen (Abschn. $6.4,6.5)$ nutzen ebenfalls identische Bauteile, erzeugen aber eine definierte Krümmung des Systems durch Falten bzw. Biegen. Die letzten beiden Arbeiten (Abschn. 6.6, 6.7) nutzen digitale Entwurfswerkzeuge, um individuelle Bauteile für eine dreidimensionale Ausformung zu produzieren.

\section{$6.1 \quad$,Form Follows Tension' von Sebastian Huth}

SEBASTIAn HUth befasste sich mit Tensegrity-Strukturen. Im Gegensatz zu den bekannten Zug- und Druckstäben wurden Biegeelemente eingeführt, die sowohl die Druckkomponente übernehmen als auch für die Vorspannung der Zugseile und Membranen sorgen. Sein Grundmodul besteht aus zwei elastisch gebogenen Federstählen, die orthogonal mit konkaver Seite zueinander stehen. Die vier Endpunkte der Bögen sind gleichzeitig Ansatzpunkte für ein Vierpunktsegel und zwei Kopplungsseile, welche jeweils die beiden Enden eines Bogens mit dem Hochpunkt des „Partnerbogens“ verbinden. Die Spannung der Membran und der Seile hält sich im Gleichgewicht und stabilisiert die Federstähle in ihrer Lage. 


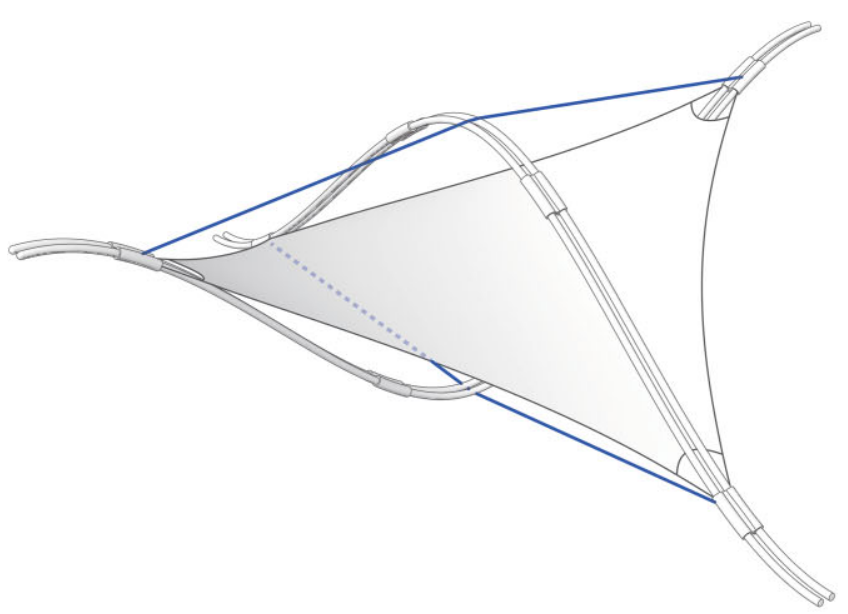

Bild 2 Tensegrity Modul mit innenliegendem Vierpunktsegel und Kopplungsseil auf Ober- und Unterseite

Tensegrity module with membrane and horizontal cables on top and bottom

Die Module können in einem orthogonalen Raster vervielfältigt werden, um ein planares System zu schaffen. Es entstehen wellenförmig gebogene Druckelemente, die sich ineinander verweben, jedoch nie berühren. In jedem Kreuzungspunkt befinden sich ein Vierpunktsegel und ein Seilpaar, die so die Lage der Wellenelemente fixieren. Das System steht unter Spannung. Der elastisch gebogene Stahl möchte sich in seinen horizontalen Ausgangszustand zurückbewegen. Membrane und Seile verhindern dies. Es herrscht inneres Gleichgewicht.

Der Ansatzpunkt der Membran kann theoretisch entlang dem Federstahl so verschoben werden, dass eine größt-

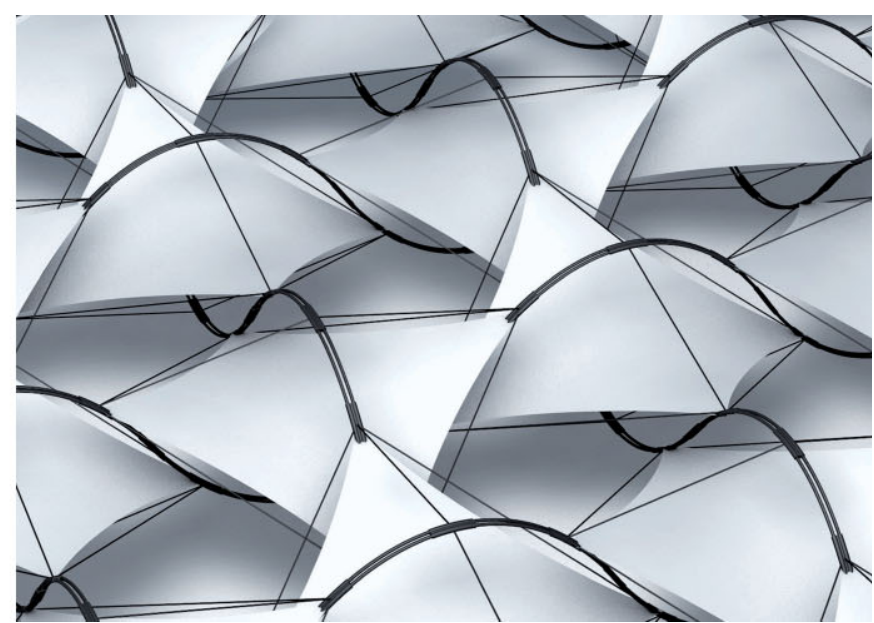

Bild 3 Verknüpfung des Einzelmoduls zu einem Flächentragwerk A single module can be added up to form a surface structure

mögliche Membranfläche und eine Überlappung der Nachbarmembranen erzeugt werden. Dennoch bildet das System keine geschlossene Haut. Abhängig vom Blickwinkel erscheint die Fläche offen oder „kariert“ geschlossen.

Um das seitliche Ausweichen zu minimieren, wurden die Federstähle paarweise versetzt aneinandergereiht. Die Kopplungspunkte konnten gleichzeitig als Anschlusspunkt für die Seile und Membranen genutzt werden. Der Membranzuschnitt wurde über ein 3D-Modell erzeugt und unter Berücksichtigung der Randschlaufen an einer CNC-Fräse zugeschnitten. Im vorgegebenen Maßstab konnte eine gleichmäßige Vorspannung der Randseile

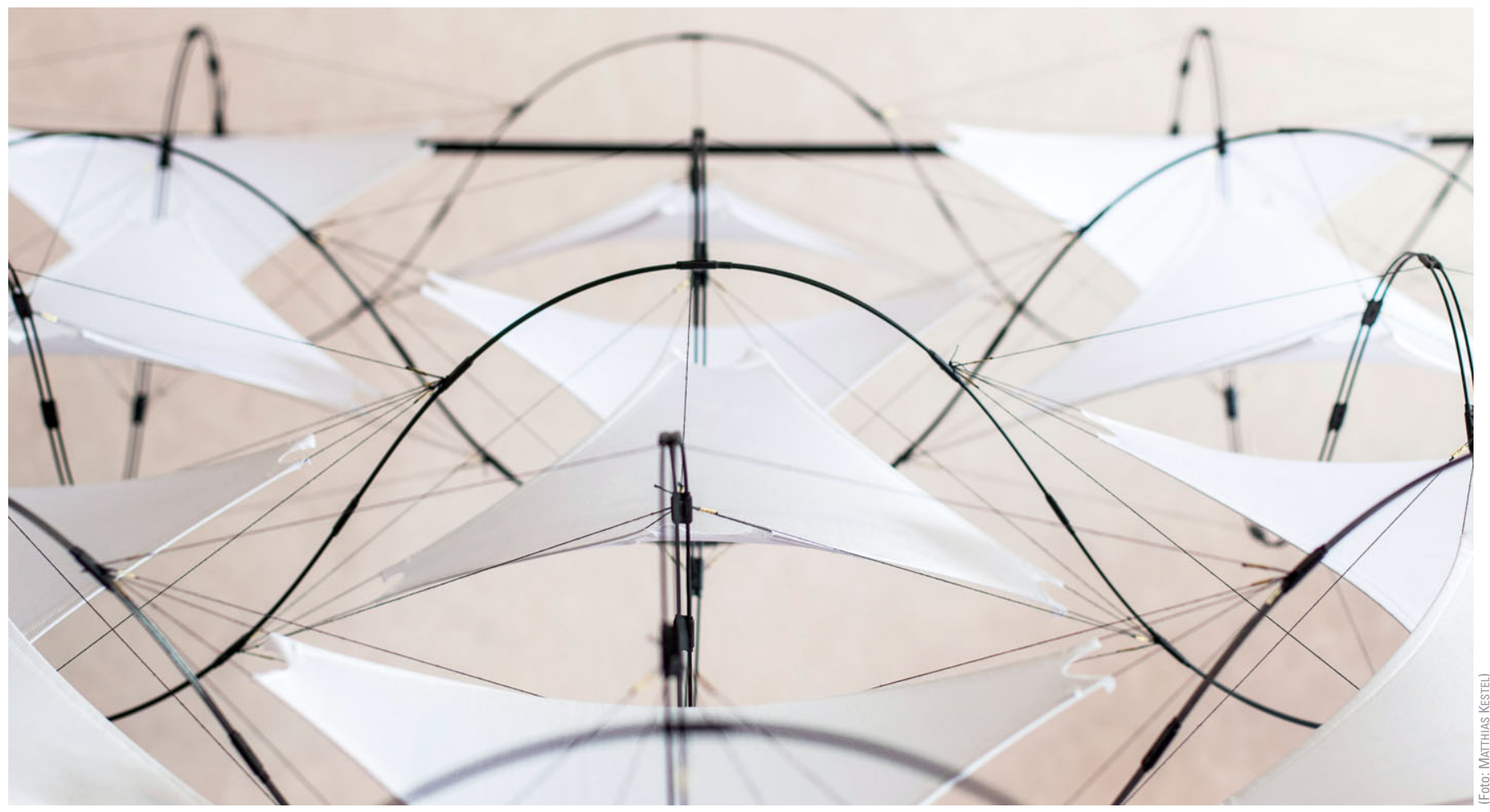

Bild 4 Tensegritystruktur aus gebogenem Federstahl, Seilen und Membranen von SEBASTIAN HUTH Tensegrity structure made of bend steel wire, cables and membranes by SEBASTIAN HUTH 


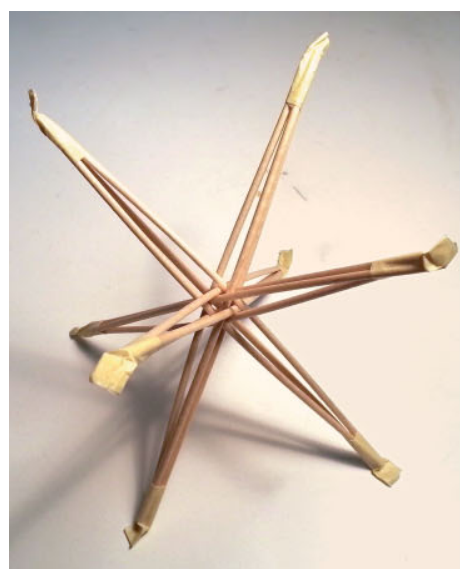

Bild 5 Räumliches Scherenmodul aus vier Stabgruppen mit jeweils drei Stäben Spacial scissor module made out of four groups of sticks

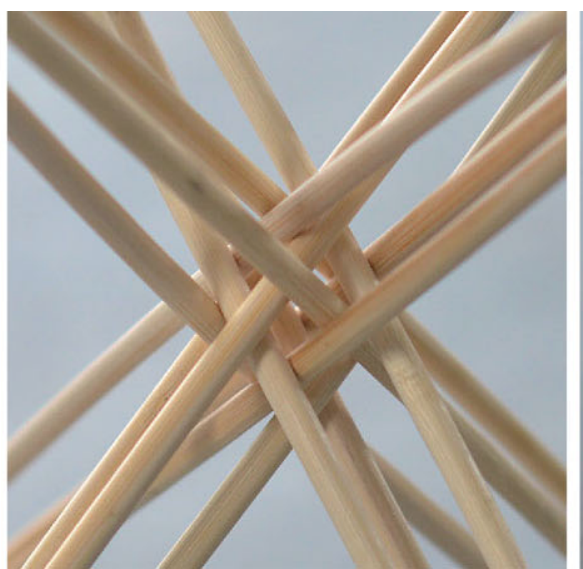

Bild 6 Geflochtener Knotenpunkt des Scherenmoduls

Braided scissor joint

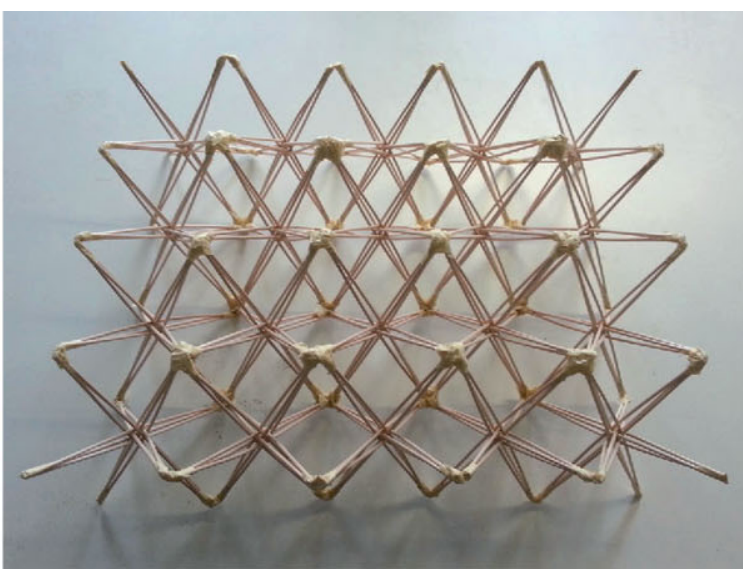

Bild 7 Werden die Module an den Stabenden gekoppelt, ergeben sie ein dynamisches System

Connecting the modules at their extremities produces a dynamic scissor system nicht garantiert werden. Es wurden daher zusätzlich Diagonalseile eingeführt, um die Eckpunkte der Vierpunktsegel zu verbinden.

Die Montage war eine große Herausforderung, da ein Großteil der Bauteile im labilen und geometrisch undefinierten Zustand verknüpft werden musste. Erst mit dem letzten Element stellten sich das Gleichgewicht und die endgültige Form ein.

Die fertige Struktur hat ein unerwartet kontrolliertes Erscheinungsbild. Die materialeigene Formensprache der gespannten Membran und des gebogenen Stahls harmo- nieren. Die Segel scheinen optisch zwischen den feinen Wellen zu schweben. Bei vertikaler Belastung greifen die unteren Seile. Das System ist erstaunlich steif.

\subsection{Deploayable Dome' von Dorian ZanK}

Durch die Verflechtung von vier Stabgruppen aus jeweils drei elastischen Stäben schuf DORIAN ZANK ein räumliches Scherenmodul. Die Stäbe wurden punktsymmetrisch aneinander vorbeigeschoben und an den Enden tangential verbunden. Die drei Einzelstäbe in jeder Gruppe werden durch den geflochtenen Knotenpunkt ge-

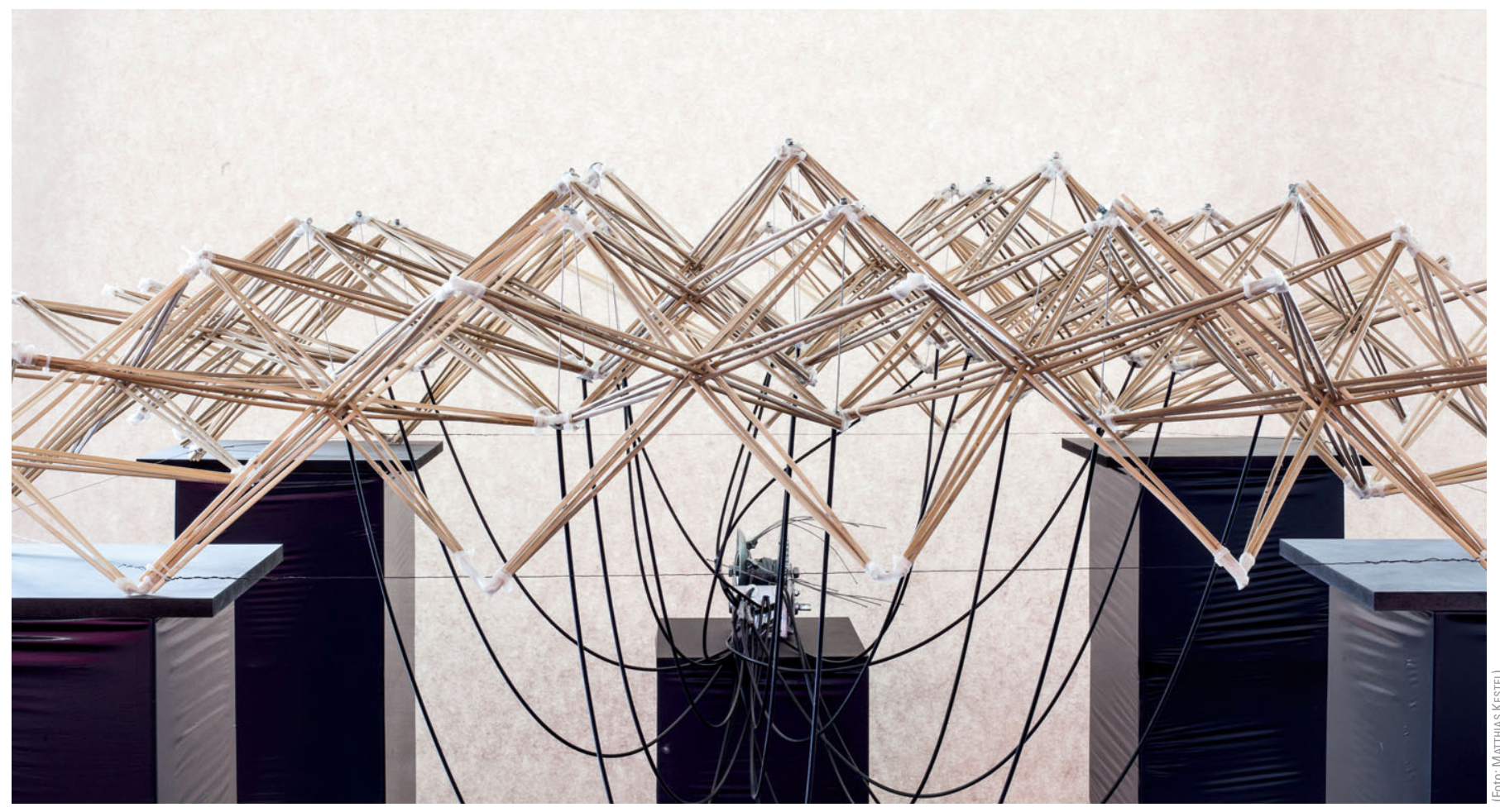

Bild 8 Die Scherenkuppel von DORIAN ZANK kann über eine Kurbel angehoben und abgesenkt werden The scissor dome by DORIAN ZANK can be raised or lowered using a hinge 


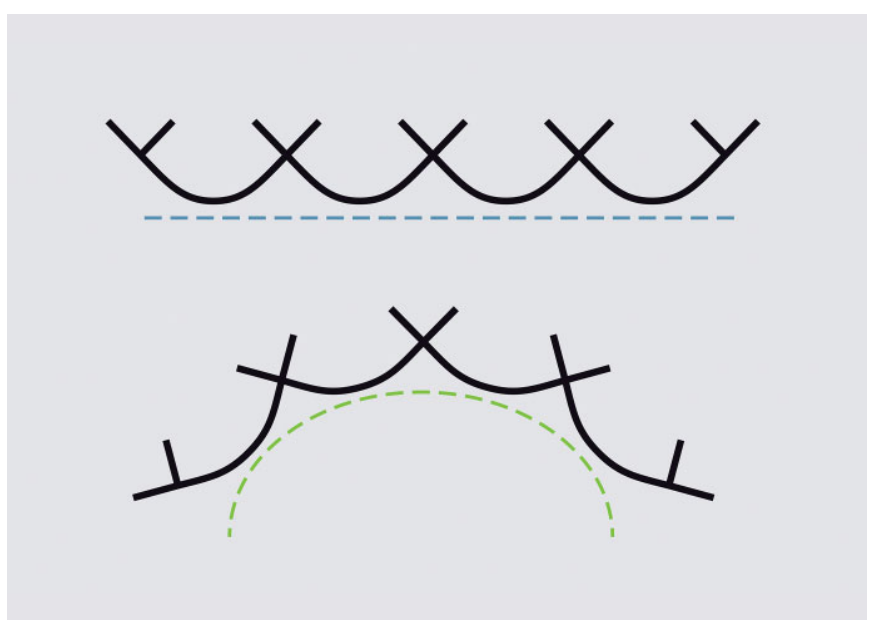

Bild 9 Durch die Kopplung von elastisch gebogenen Elementen entsteht Spannung, die das System nach oben drückt

Linking elastically bent elements causes stress, which pushes the system upward

spreizt und erzeugen dadurch Reibung. Die Stabgruppen bilden ein räumliches Gelenk, das eine symmetrische Scherenbewegung zulässt.

DORIAN ZANK verwendete einfache Bambusspieße und verknüpfte 36 Module zu einem quadratischen System mit $6 \times 6$ Einheiten. Die Scheren wurden an ihren Endpunkten mit flexiblen Schläuchen und Kabelbindern gelenkig verbunden. Das resultierende Scherensystem ist beweglich. Es kann zu einem kompakten hohen Bündel zusammengezogen oder zu einem weiten flachen Rost ausgedehnt werden.

Ziel war es, diese Flexibilität in eine bewegliche Kuppel $\mathrm{zu}$ übersetzen, die von einem zentralen Punkt gesteuert

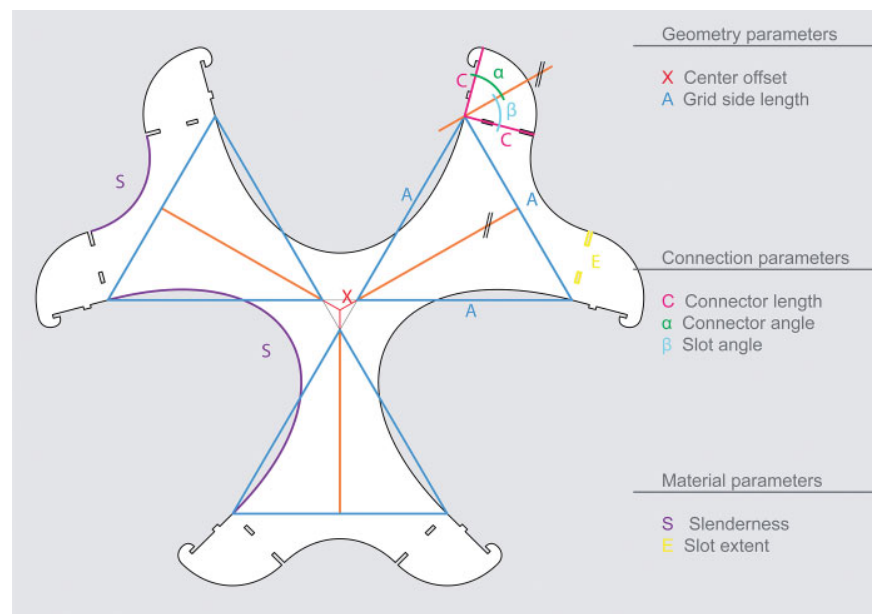

Bild 10 Das Einzelmodul ist auf einem Hexagon aufgebaut; die Proportionen steuern die Biegespannung im geknüpften Zustand

Single module based on a hexagon; the bending stress in the system can be regulated by changing the proportions

werden kann. Dafür wurden zwei Zugelemente eingeführt. Ein Ringseil entlang der unteren Außenkante definierte eine maximale horizontale Ausdehnung des Systems. Die übereinanderliegenden Knotenpunkte wurden mit vertikalen Zugseilen verbunden. Diese spreizen die Scheren und damit das Gesamtsystem gegen das Ringseil. Da das System sich horizontal nicht weiter ausdehnen kann, drückt sich der Stabrost kuppelartig nach oben.

Für die Zugseile wurden einfache Fahrrad-Bremskabel verwendet. Um die Bewegung der Kuppel zentral zu steuern, wurden alle Bremskabel durch Druckschläuche zu einer Kurbel weitergeführt. So können die Zugseile gleichzeitig und identisch verkürzt werden. Mit

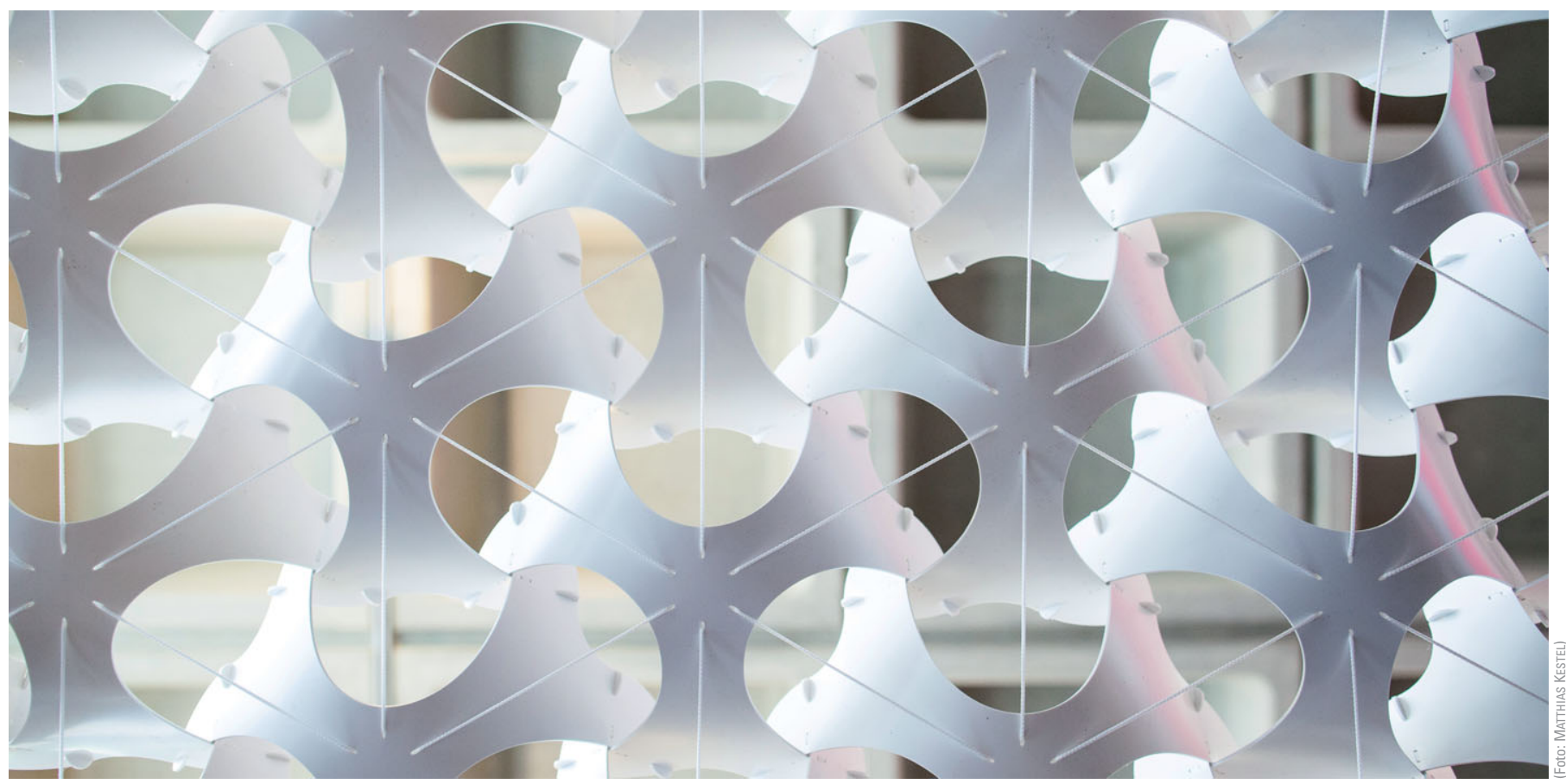

Bild 11 Untersicht der hexagonalen Biegestruktur von QUIRIN MÜHLBAUER

View from below of the hexagonal bending structure by QUIRIN MÜHLBAUER 
jeder Drehung wird die Kuppel weiter nach oben gespreizt.

In diesem Projekt wurde durch herkömmliche Materialien und Verbindungen eine komplexe Mechanik verwirklicht. Die Einfachheit des Scherengelenks, in dem sich die Stabgruppen verflechten und nach außen hin wie ein Kragträger verjüngen, trägt zu einer außergewöhnlichen Ästhetik dieser mobilen Kuppel bei.

\section{3 ,Hexagonal Bending' von QUIRIN MÜHLBAUER}

Wie kann man sich die Spannung in einem elastisch gebogenen Bauteil zunutze machen? Diese Frage stellte sich QUIRIN MÜHLBAUER bei der Entwicklung seiner Wabenstruktur.

Ausgangspunkt war die lineare Kopplung von gebogenen Elementen. Durch die innere Spannung wird das System nach oben gedrückt, sobald man Anfang und Endpunkt fixiert. Wendet man denselben Gedanken auf eine Fläche an, ergibt sich ein geschlossenes System aus elastischer Spannung im Inneren und Zugspannung im Rand. Die Skulptur weicht nach oben aus, ohne an den Auflagern fixiert werden zu müssen.
Auf einem hexagonalen Raster aufbauend wurde ein ebenes sternförmiges Grundmodul entwickelt. Dieses besteht aus drei konzentrischen Armen, die sich verzweigen und so sechs Verbindungspunkte ausbilden. An jedem Verbindungspunkt schließen über eine biegesteife Verzahnung zwei weitere Module an. Die Verbindung wirkt als Einspannung und biegt das Modul in eine Kelchform. Es entsteht ein wabenförmiges Gesamtsystem aus biegesteif verschränkten dreiarmigen Kelchen, die auf der Oberseite gekoppelt sind und auf der Unterseite eine bauchige Form ausbilden. Das System erhält so eine statische Höhe, die durch ein Seilnetz auf der Unterseite aktiviert werden kann. Die Unterspannung verleiht dem System die zusätzliche Steifigkeit, die für die Skulptur aus $2 \mathrm{~mm}$ dünnen Polysterolplatten bei ca. $2 \mathrm{~m}$ Spannweite nötig ist.

Bemerkenswert ist die leichte kuppelartige Überhöhung, die sich durch die elastische Spannung einstellt, sobald die Module verknüpft werden. Die punktsymmetrische Verzahnung der gebogenen Kelche hat den Anschein einer optischen Täuschung wie in Grafiken von M. C. Escher. Durch die Öffnungen wird das Licht indirekt nach unten reflektiert. Das Seilnetz wirkt wie ein Koordinatensystem, das die gebogenen Formen ordnet.

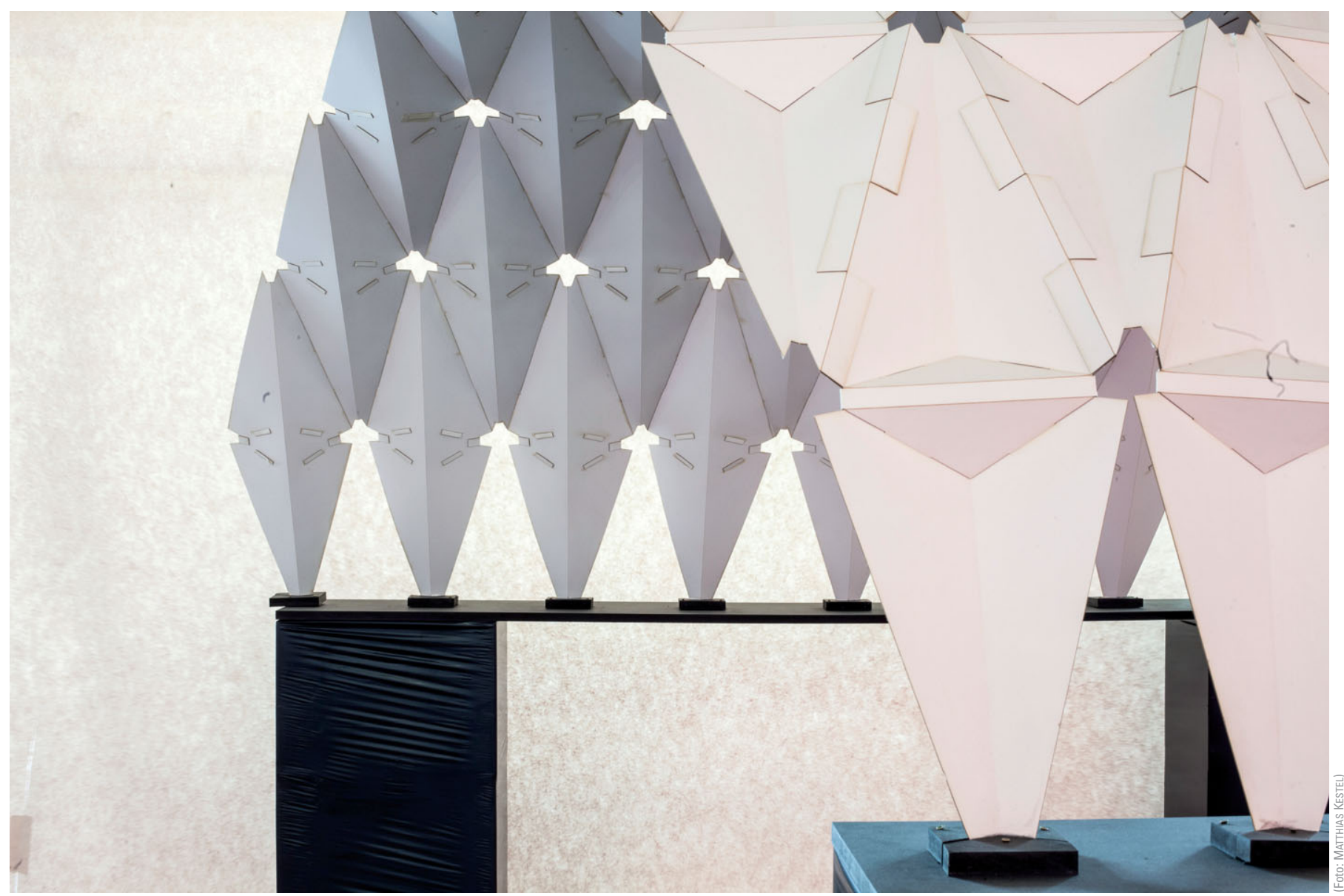

Bild 12 Rautenfaltwerk von MiRSOLAVA DENINA

Diamond folding structure by MirSOLAVA DENINA 

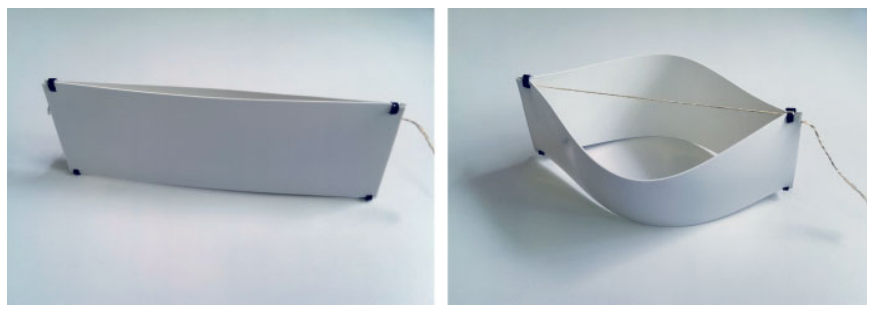

Bild 13 Zwei Bänder werden mit einem Seil zusammengezogen und erzeugen eine rautenförmige Wabe

Two ribbons being pulled together by a tension rope to form a diamond shape cell

\section{4 ,Folding Wing' von Miroslava Denina}

Miroslava Denina beschäftigte sich mit Faltstrukturen. Sie definierte eine Raute mit den Proportionen $3: 5$ und faltete diese entlang der langen Mittelachse. Durch die Verknüpfung mehrerer Module entlang den Kanten entstand ein einfach gekrümmtes Rautenmuster gleich einer Tonnenschale. Alle Module sind identisch. Die Gesamtform ist von dem Faltwinkel der einzelnen Module abhängig. Ist die Faltung flach, entsteht ein breites System mit leichter Krümmung. Erhöht man die Faltung, zieht sich das System in Querrichtung zusammen. Gleichzeitig erhöht sich die Krümmung der Tonne.
Das System ist wie eine Ziehharmonika über die gelenkigen Kantenverbindungen beweglich. Um die $2 \mathrm{~m}$ weit gespannte Skulptur auszusteifen, wurde ein Element in Querrichtung eingefügt. Diese kleine, ebenfalls gefaltete Raute folgt der Logik des großen Moduls und kann als Verbindungsstück zwischen dessen Flächen gesteckt werden. Beide Rauten stabilisieren sich so gegenseitig. Es entsteht eine horizontale Gliederung auf der Außenseite. Die kreuzförmigen Öffnungen in den Knotenpunkten erleichtern die Montage und schaffen regelmäßige Lichtpunkte in der Untersicht.

\subsection{Bending Strips' von David Walsh}

DAVID WALSH untersuchte die Verhaltensweise von elastischen Bändern. Das Modul besteht aus zwei gekoppelten Polysterolstreifen, die mit einem innen liegenden Seil zusammengezogen werden. Dies hat zur Folge, dass die Bänder sich nach außen biegen und eine geschwungene rautenförmige Wabe bilden.

Positioniert man das Drahtseil nicht mittig, sondern exzentrisch an der Oberseite der Elemente, erhält die Wabe zusätzlich eine Krümmung. Dabei stellt sich ein weiterer geometrischer Effekt ein. Je weiter sich die Wabe biegt (also je mehr das System vorgespannt wird), umso mehr

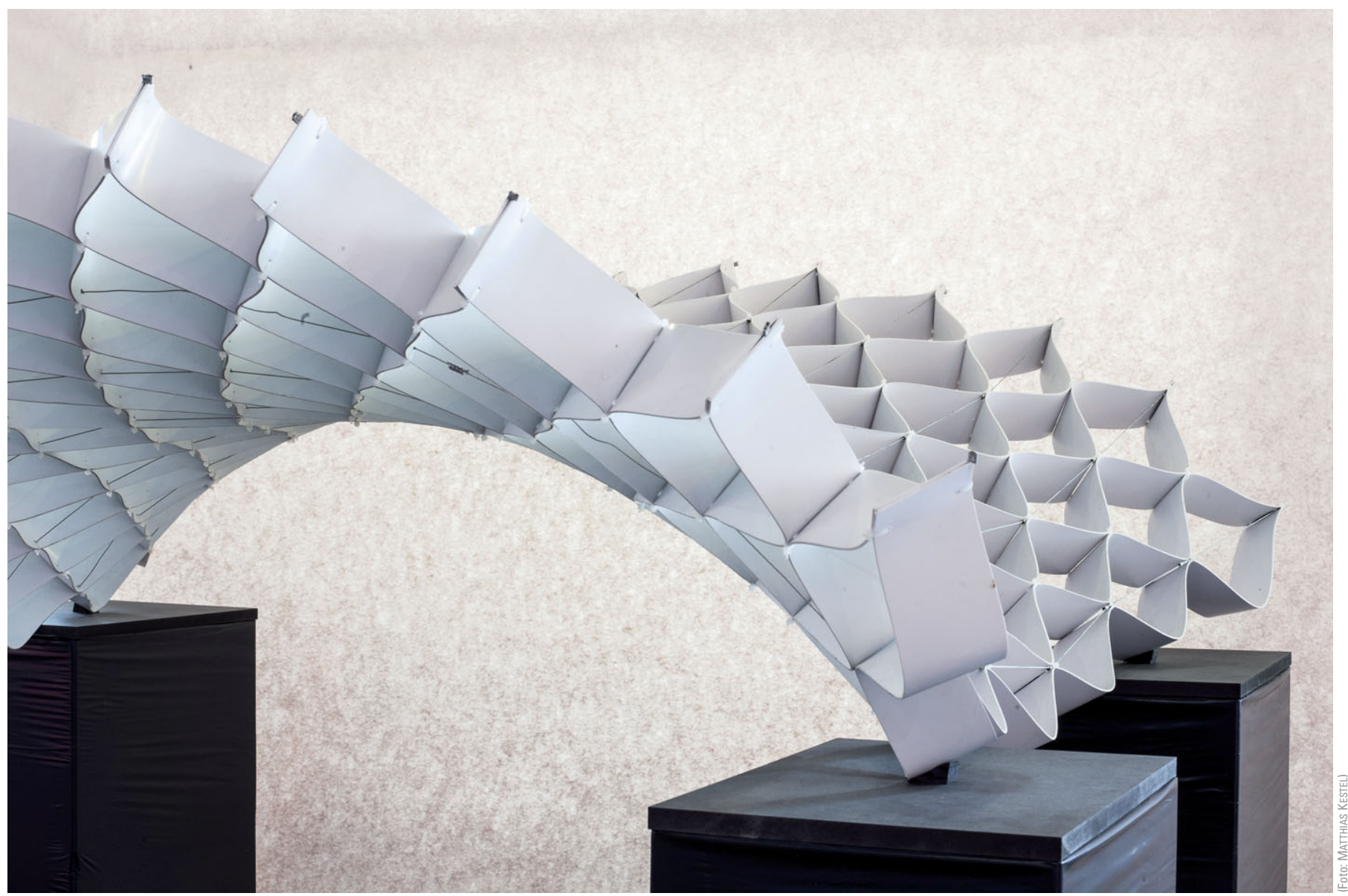

Bild 14 Sattelfläche aus gebogenen Polysterolstreifen von DAVID WALSH Saddle shaped shell out of bend strips of plastic by DAVID WALSH 
wandern die obere Elementränder auseinander. Die Bänder erfahren eine Torsion, und die Wabe öffnet sich trichterförmig.

Um das Modul zu vervielfachen, werden lange, gleichförmige Polysterolstreifen ausgelegt und mit Kabelbindern abwechselnd mit den benachbarten Streifen verknüpft. Durch die Schlaufen der Kabelbinder werden Drahtseile gezogen. Die Seile werden vorgespannt und dann an jedem Kabelbinder mit einer Aluminiumhülse verklemmt. Dadurch wird sichergestellt, dass alle Rauten gleichförmig bleiben. Die einseitige Krümmung jedes Moduls addiert sich in der Gesamtskulptur zu einem weiten konkaven Schwung. Die Trichterform der Waben hingegen erzeugt eine Gegenkrümmung in Querrichtung. Es entsteht eine Sattelfläche.

Die Geometrie der Gesamtstruktur ist direkt an die Proportionen der einzelnen Wabe gekoppelt. Das System lässt also keinen Spielraum für entwerferische Freiheiten. Die gegensinnige Krümmung der Skulptur ist dennoch ausgewogen und wird durch das Schattenspiel der gebogenen Wabenstruktur belebt. Die Schalenform schafft eine einladende Torsituation für Besucher.

\subsection{Cubic Surface' von Michal MarkuSeK}

Michal MARKUSEK legte als sein Grundmodul einen $10 \times 10 \times 10 \mathrm{~cm}$ großen Würfel fest. Die 153 Einheiten sind als dreidimensionaler Vierendelrahmen aus 2-mmMDF-Platten gefräst und verleimt. Aneinanderliegend ergeben die Würfel ein $9 \times 17$ Einheiten großes planares System.

Ziel war nun, durch variierende Verbindungen zwischen den Kuben Krümmung in zwei Richtungen zu erzeugen. Es wurden ein minimaler und ein maximaler Abstand

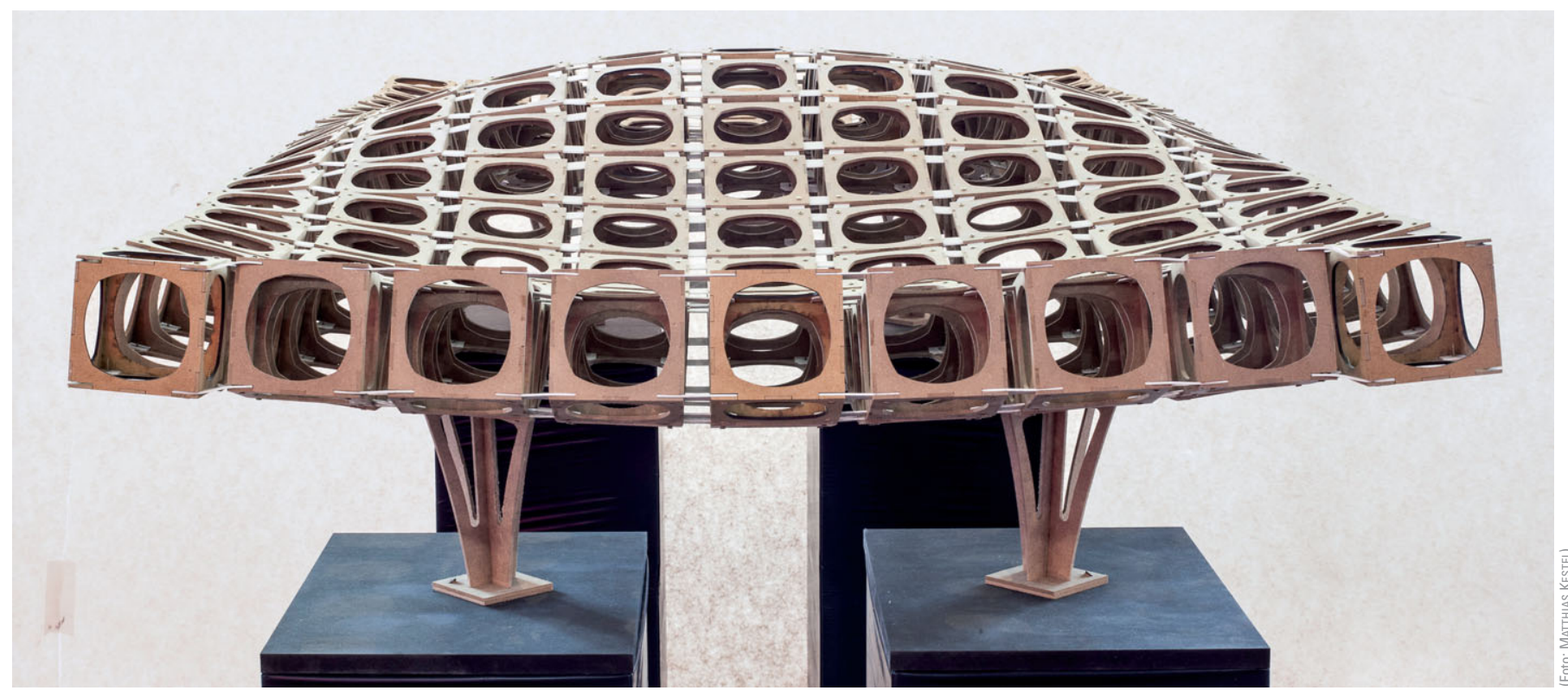

Bild 15 Zweifach gekrümmte Oberfläche aus regelmäßigen Kuben von MICHAL MARKUSEK Cubes form a double curved surface by MICHAL MARKUSEK

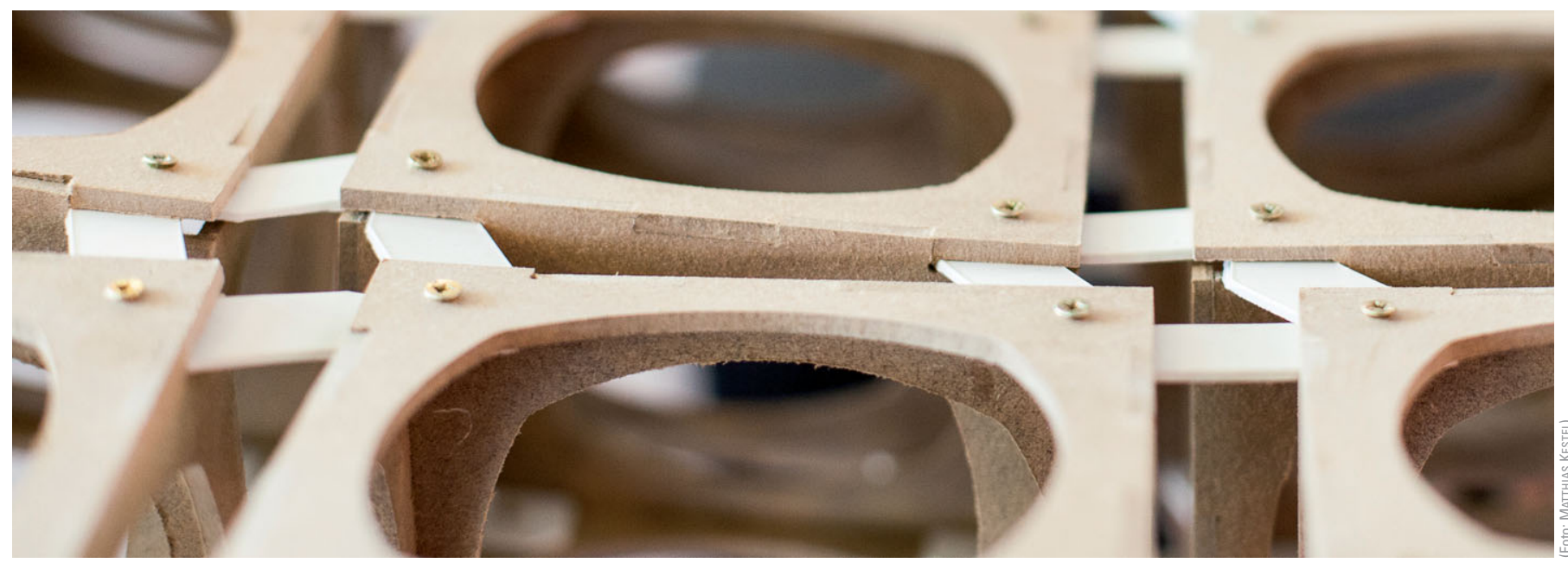

Bild 16 Die Würfel werden durch individuelle Ringe aus Polysterol verbunden The cubes are connected by individual Polysterol elements 

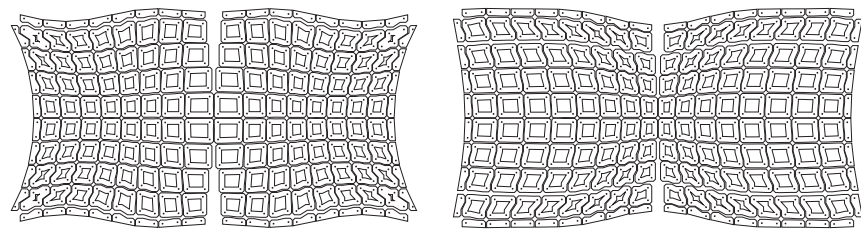

Bild 17 Die Verbindungselemente der Oberseite (links) und Unterseite (rechts) ergeben ein flächenfüllendes Muster

The connection rings on top (shown left) and bottom (shown right) can be laid out to form a continuous pattern

zwischen zwei Würfeln festgelegt und daraus die möglichen Krümmungsradien der Skulptur berechnet.

Der Planungsprozess wurde in einer parametrischen Designsoftware durchgeführt. Eine Freifläche mit einem Hochpunkt und zwei symmetrischen „Tälern“ wurde anhand der vorgegebenen Krümmungsradien entworfen. Auf dieser Oberfläche wurde ein Punkteraster generiert, das im zentralen Hochpunkt der Fläche fixiert ist und sich in alle Richtungen in regelmäßigen Schritten von $12 \mathrm{~cm}$ ausdehnt. Die so ermittelten Punkte bilden die Mittelpunkte der Würfel, die tangential zur Oberfläche platziert sind. Durch die 2-cm-Fuge zwischen den Einheiten haben sie den Freiraum, sich der Neigung der Oberfläche anzupassen, ohne mit ihren Nachbarn zu kollidieren.

Durch die genaue Positionierung der Würfel im Raum konnten deren Abstände an Ober- und Unterseite ausgelesen werden. Dabei stellte sich heraus, dass das kubische System sich nicht nur durch eine Winkeländerung an die Oberfläche anpasste. In den Eckbereichen ergab sich zudem ein Versatz in horizontaler und vertikaler Richtung.

Als Verbindungsmittel wurden insgesamt 352 individuelle Elemente aus 1,5 mm starkem Polysterol zugeschnitten, die seitlich in die Kuben geschoben und von oben bzw. unten verschraubt wurden. Durch manuelle Verformung konnten diese Ringe den vertikalen Versatz aufnehmen und dennoch eine starre Verbindung erzeugen. Die Ausdehnung des Systems ist in beide Richtungen begrenzt, da der Versatz zwischen den Kuben ab einer bestimmten Entfernung vom Zentrum zu groß wird, um noch eine ausreichend stabile Verbindung der Würfelecken zu verwirklichen. Die 2D-Abwicklung der Verbindungsstücke wurde über die Software ausgelesen und mit der CNCFräse gefertigt. Dabei fiel eine überraschende Eigenschaft auf: Die rautenförmigen Verbindungselemente konnten flächenfüllend wie ein symmetrisches Puzzle ausgelegt werden. Die Eckwinkel vier benachbarter Ringe addieren sich zu $360^{\circ}$.

Der entstandene Vierendelkörper ist weniger wegen seines Tragverhaltens als vielmehr wegen der lehrreichen geometrischen Darstellung von akademischem Wert. Der Kontrast zwischen den starren Kuben und den in sich gewundenen Verbindungselementen veranschaulicht deutlich die sukzessive Auswirkung einer zweifach gekrümmten Fläche auf ein orthogonales Raster.
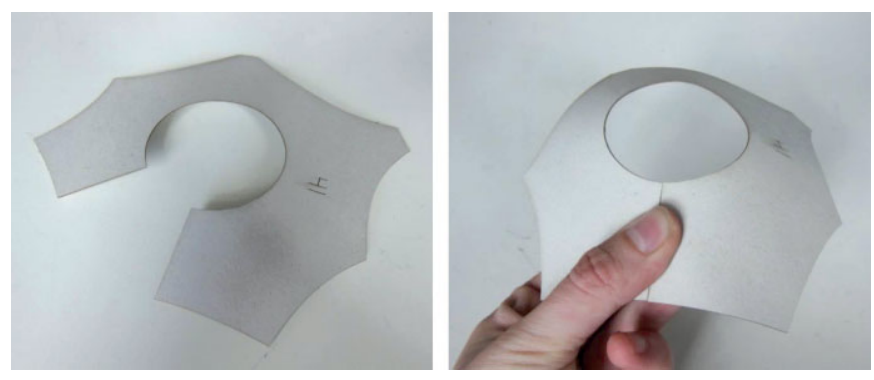

Bild 18 Kegelstumpf im Zuschnitt und im gebogenen Zustand Truncated cone as cut-out and bent into shape
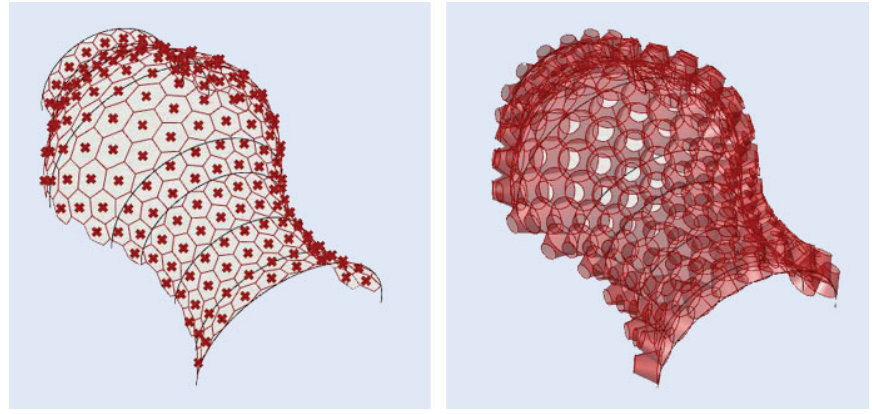

Bild 19 Designoberfläche mit hexagonalem Punkteraster und sich überlagernden Kegelstümpfen

Design surface with hexagonal point grid and overlapping truncated cones

\subsection{Modular Shell' von Vitaly EnTIN}

Die präzise Darstellung von elastisch gebogenen Bauteilen stellt nach wie vor eine Herausforderung für digitale 3D-Modelle dar. VITALY EnTIN suchte daher eine Biegeform, die sich einfach am Computer modellieren und berechnen lässt. Seine Wahl fiel auf den Kegelstumpf.

Mithilfe einer 3D-Software entwarf er eine zweifach gekrümmte Designfläche - ähnlich einem Iglu mit Torbogen und anschließender Kuppel - und generierte darauf ein hexagonales Punkteraster. In jedem Punkt wird automatisch mithilfe eines Computeralgorithmus ein Kegelstumpf erzeugt, der nach außen gerichtet ist. Die Höhe und die Radien des jeweiligen Kegelstumpfes sind von der Krümmung der Designfläche an dieser Stelle und dem Abstand zu den benachbarten Punkten abhängig. Die Kegelstümpfe wurden so programmiert, dass sie sich mit ihren sechs Nachbarn verschneiden. Wendet man diese Schnittkurven auf die Kegel an, so bleibt ein Kegelstumpf mit sechs geschwungenen Anschlussbögen.

Die Abwicklung der insgesamt 171 unterschiedlichen Kegelstümpfe wurde ausgelesen und für die weitere Verarbeitung flächig ausgelegt und nummeriert. Die gesamte Struktur wurde mit dem Lasercutter aus 1,5-mm-Polysterolplatten gefertigt. Die Schnittkanten wurden verzahnt und an den Hoch- und Tiefpunkten durch Kabelbinder verbunden. Durch die Höhe der Schnittkurven kann im Verbund eine ausreichende Biegesteifigkeit erzeugt werden. 


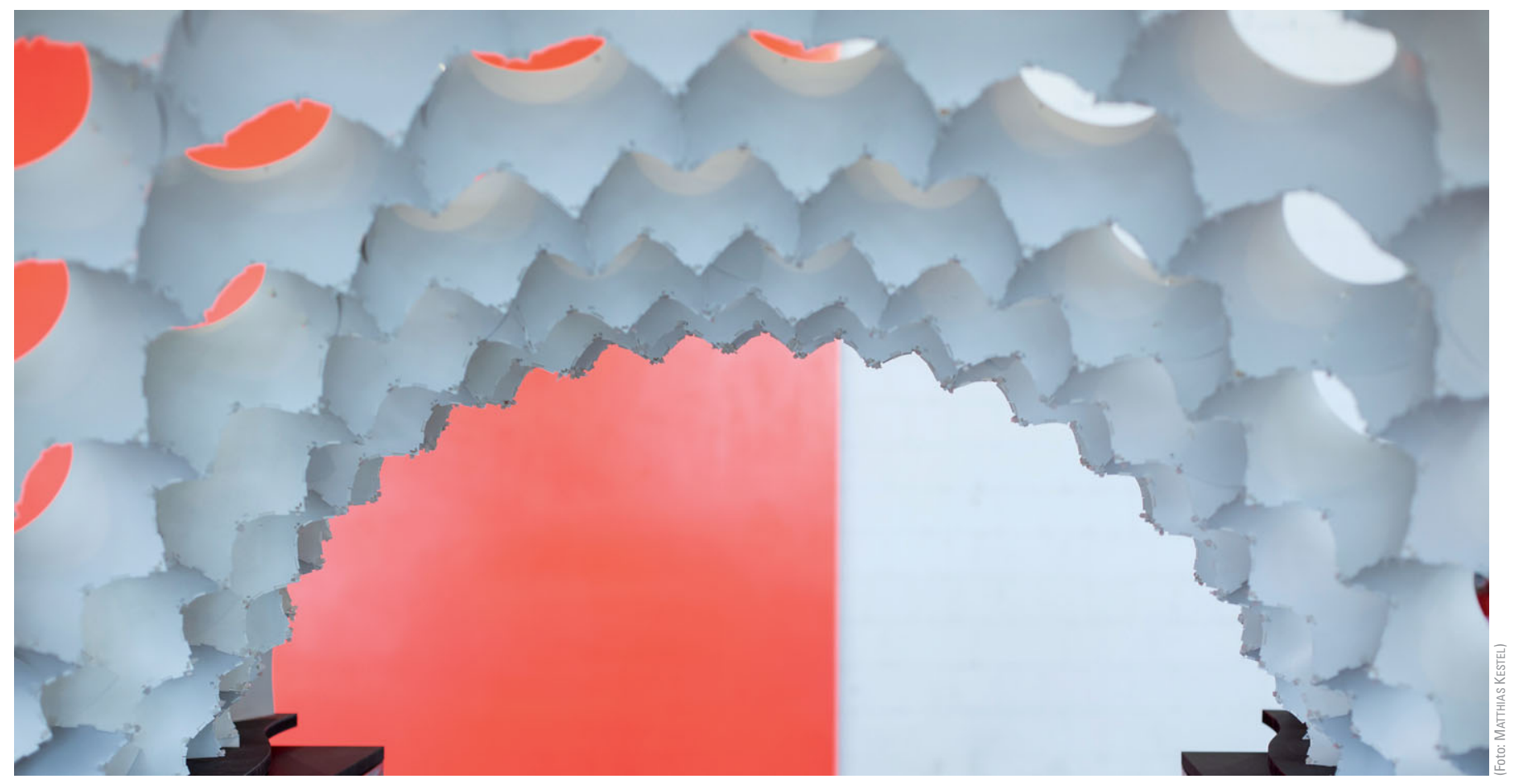

Bild 20 Untersicht der modularen Schale aus gebogenen Kegelstümpfen von VITALY ENTIN View from below of the modular shell by VITALY ENTIN

Es entsteht eine fein aufgelöste Schale, auf der sich das hexagonale Raster noch gut ablesen lässt. Im Inneren der Skulptur nimmt man die Kegelstümpfe als fast sphärische Bullaugen mit scharfen Schnittkanten wahr. Auf der Außenseite erinnern die homogenen Kreisöffnungen an das Tragskelett von Seeigeln.

\section{7}

\section{Fazit}

Für eine Symbiose zwischen Formgebung, Herstellung und Tragwirkung wurden unterschiedliche Lösungsansätze gefunden. Dabei wurde eine Vielfalt an selbsttragenden modularen Freiflächen erzeugt, die durch zweidimensionale Herstellungsmethoden und mit gleichen Bauteilgruppen gefertigt werden konnten.

Anhand der gebauten Skulpturen konnte die Tragwirkung für das Eigengewicht in kleinem Maßstab veranschaulicht werden.

In den kommenden Semestern soll überprüft werden, inwieweit sich diese Ansätze auf einen architektonischen Maßstab übertragen lassen. Für welche Nutzung oder Funktion lassen sich die neu gefundenen Strukturen anwenden? Können die Tragsysteme für eine angemessene Spannweite eingesetzt werden? Es wird eine Weiterentwicklung bis hin zur Verwirklichung eines funktionalen Pavillons angestrebt.

\section{Projektbeteiligte}

Prof. Dr.-Ing. RAINER BARTHEL

Dipl.-Ing. Architekt EIKE SCHLING

Lehrstuhl für Tragwerksplanung

Technische Universität München

Studenten (Master):

Miroslava Denina, Vitaly Entin, Sebastian Huth, Michal Markusek, Quirin MüHLBAUER, DAVID WALSH, DORIAN ZANK

\section{Autoren}

Dipl.-Ing. Architekt Eike Schling

Lehrstuhl für Tragwerksplanung, Architekturfakultät

Technische Universität München

Arcisstr. 21

80333 München

eike.schling@tum.de

\section{Prof. Dr.-Ing. Rainer Barthel}

Lehrstuhl für Tragwerksplanung, Architekturfakultät

Technische Universität München

Arcisstr. 21

80333 München

r.barthel@|rz.tu-muenchen.de

Dipl.-Ing. Joram Tutsch

Lehrstuhl für Tragwerksplanung, Architekturfakultät

Technische Universität München

Arcisstr. 21

80333 München

joram.tutsch@tum.de 